\title{
Research of Car Navigation Data Retrieval System
}

\author{
http://dx.doi.org/10.3991/ijoe.v9iS4.2596 \\ Xiaokui Ren, Xiujuan Li and Lirong Ma \\ Liaoning Technical University, Huludao, China
}

\begin{abstract}
In recent years, the automotive industry has become a pillar industry of the world, the role played by the automobile in the daily life and work has become increasingly prominent. In the car navigation retrieval system, whether from the perspective of retrieval speed, screen display, or memory management, it can not meet the needs of users. In the design, the retrieval algorithm along the road of facilities retrieval can not only improve the retrieval system's modular structure, but also introduce the idea of thread pool. The whole system is divided into four modules; B-Tree + phenotype data structures being used can improve the search speed; a new road merging algorithm being proposed can improve the retrieval performance.
\end{abstract}

Index Terms-Retrieval algorithm along the road; thread pool; GPS; B-Tree+

\section{INTRODUCTION}

With the increase of car population, the concept of ITS comes into people's view. The combining of the communication technology, electronic control technology, information technology and control technology are applied into transportation management effectively. The contact between roads, vehicles and drivers is established using modern science and technology. The car navigation figures that construction projects currently carried out by a variety of intelligent transportation systems can be captured $^{[1,2,3]}$.

Mainstream product for the central navigation is the unified navigation and it can navigate with real-time traffic monitoring system through the ground. A 24h GPS of the $3 \mathrm{D}$ position of the vehicle. It is providing travel vehicles with the optimal path to reach the destination. It has accurate path planning, fast speed of calculating the path, it also can display on the E-map and provide the running method at the same time. And provide the users with the vehicle or surrounding locations, hotels, hospitals, and other information of a specified location, and show its location on the map ${ }^{[4,5,6]}$.

The problem of navigation products on the market, in short, the most outstanding performance is data retrieval system. In the field of navigation technology research and development, the E-map and the facility search collectively are called a data retrieval system. They are realized through the retrieval of the data file, whose facilities retrieval is the retrieval of data files. To find out the location details of the users, catering services, gas stations, hotels and other facilities are needed, and make the list displayed on the screen. E-map is ordered retrieval of the data file and parse the data information will eventually be drawn out on a display screen. And navigation data retrieval fault mainly has the following several aspects ${ }^{[7,12,13]}$ : Facilities retrieval speed is too slow; data file format is not flexible, which cause the program take too much time to locate; Facilities retrieval can only achieve to own car or destination as the center to search facility places at present, the function is too thin; 3D E-map display is too largely, and the text deformation is more serious distortion.

\section{NAVIGATION DATA RETRIEVAL System}

Data retrieval system is the core of the navigation module. Its most notable feature is dependent on the data. Fig.1 shows the structure of the data retrieval. The data retrieval system comprises the two modules of the facility search, and the E-map. Searching facilities complete the parsing of the data information and made a list displayed on the screen eventually through the retrieval of the data file. E-map is the retrieval of data files parsing the data in accordance with the pattern classification layered painted screen.

Facilities retrieval has lots of kinds, and they can divided into facilities classification retrieval, peripheral retrieval, pinyin retrieval, keyword search, domicile retrieval, etc. All kinds of retrieval process are generally the same, and the difference lies in the process of implementation. Facilities retrieval algorithm is simple in summary, first get the needed data from a storage device retrieval, and create a series of results index data list on basis of ListIndex; The second step is continuing to search List information content in line with ListIndex completed before according to the production, which also is the need of the retrieval results; The third step is when a user need to know the detailed information, the program will retrieval again and will read all of the information displayed on the screen.

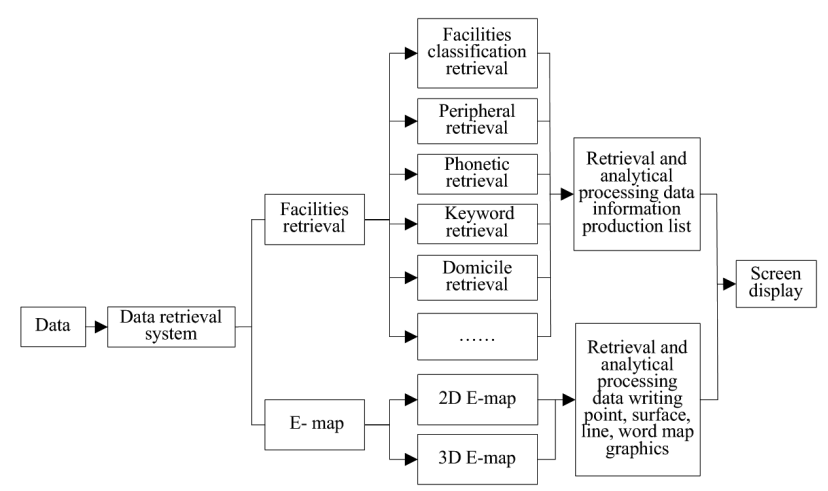

Figure 1. Structure of data retrieval system 


\section{DESIGN OF RETRIEVAL AlgORITHM}

\section{A. Improved NOTE Architecture}

Retrieval system generally called NOTE. NOTE has the complex internal structure. In order to make the code clearer, NOTE also uses hierarchical modular design thought ${ }^{[8,9,10]}$. Before the development, NOTE is divided into two parts, the first part is used to deal with retrieval process, it contains different retrieval function. The other part is used to deal the monitoring work about media. UI provides API function through the first part, and it is responsible for returning the retrieval results of NOTE meanwhile.

In order to make each function of NOTE divide clearer, designing the modular structure of NOTE. As shown in Fig.2. In the NOTE, Layer01 is in the "top" layer, it is the only external interface of NOTE, all the functions provided to the outside must be provided to the outside through Layer01. Layer02 calls the lower Layer interface (if necessary, you can also call the other Component interface), which achieves or supports the function of the Layer01. Layer02 is the core of NOTE controlling and scheduling. Layer03 achieves the function of NOTE, Layer03 carries out all searches related functions and Mark related functions. Layer99 is the common function library of NOTE, which providing NOTE other Layer with file operational function, data accesses function, analytic function, transition function and ranking function. Setting up a independent Layer99 aim.

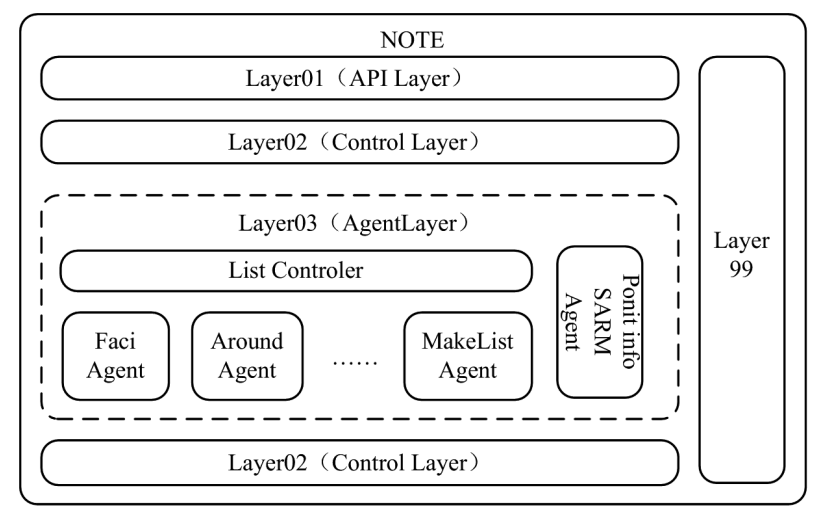

Figure 2. NOTE structure before improvement

\section{B. Improvement of Retrieval Data Format by The Road}

The China plate is divided into $\mathrm{n}$ parts with the same size according to the location; width and length are $10 \mathrm{~km}$. The center coordinates of each mesh file is the unique identification of this Mesh files, called Mesh ID. Mesh ID is the basis of the mesh to retrieve it. But many shortcomings of the storage as mentioned above, positioning speed is too slow with retrieval, by traversal to position Mesh files and after finding the corresponding Mesh, the speed of filtering the meeting-conditions is very slow.

In order to overcome the shortcoming of storage as mentioned above, B-Tree and phenotypic data structure can be used. B-Tree storage management information index structure, phenotype is used to store data. The starting position of the data blocks that fall into this category, and the total number of records to be able to look through category management information. In accordance with this format, data can be read directly, and then be screened according to the retrieval condition by distance; the retrieval speed there will be greatly improved.

Firstly, data is stored by block using blockset, block, and parcel for the unit. The national data as a large rectangular block, this rectangle is called mesh file, the mesh data is divided into $16 \times 16$ blockset averagely, each blockset is divided into $8 \times 8$ block averagely, each block is divided into $32 \times 32$ Parcel.

Secondly, all the data part of the index are stored together, the data portion is stored together, using the position of the index positioning data. Along the road facilities category includes services catering services, shopping services, auto services, accommodation services, sports and leisure, public facilities, transportation facilities, education and culture and life. Each classification following contains a small class. Management part of information store the category data offset and record size.

Finally, Retrieve data file is divided into three parts. The name of the text string length is not fixed, and can not be precise positioning, so the name of the facility is to separate out all exist in the name file. Telephone, postal code and detailed information are also isolated from a file, called pshare. The file is stored in binary form, which name and pshare file named after the AreaCode. Such as Beijing, all the details are stored in the pshare11.dat file, the facility name stored in name11.dat file. Block data is named using the number in the blockset. For example, the 32nd block in the blockset56 file named block32.dat.

Block data file record the offset the name of the facility in the pshare, besides storage more additional information, phare also contains the position of the facility name offset in the name file. The whole idea is, through block file to find more detail information in pshare file, through pshare file to research name string in name file.

Where, KiwiCode stored in entity data is the identifier of facility category, which can be used to filter facility classification; background point coordinates are latitude and longitude coordinates of the location of the facility, through its facilities and car route to calculate the distance (i.e., point to the straight-line distance); pcshare offset refers to the offset of this facility telephone, zip code, etc. The location of the detailed information stored in the file of pshare through it to locate and read the data; AreaCode is facility area code and can locate the phare file name and name file by it.

\section{Retrieval Algorithm Along Road}

As the basis of along road is a pathway, but what NOTE read from data module is a point set that, following to merge roads according to this point set? Road Mergering Algorithm, AutoNavi Holdings Limited have requested patent: firstly, to get road link which the road is not merge with other road, from road cross connection point information, then to get road link which angle is greater than 90 and no more than 180, this road link regarded as the mergering road link.

It is not perfect to merger the road through angle, because different road attributes can be mergered too. This paper propose a new algorithm, that to judge road attributes with two intersecting roads triangle features combined and to merger roads. Non-speedway attributes include National and provincial highways, city streets and general Road, fairway, little streets; highway attributes 
refer to speedway (including Ramp Road), urban fast$\operatorname{track}^{[11]}$.

Define: isExpresswayPre and isExpresswaySrch are used as curse to judge road attributes. Initial curse attribute, if the first-segment road is highway or fast-track, then let isExpresswayPre=1; isExpresswaySrch=1; If the forward segment link is not a highway or fast-track, let isExpresswaySrch=0; isExpresswayNext=0; If it is the second segment link. If the forward segment link of car is highway or fast-track, then isExpresswayNext=1; if the forward segment link of car is not highway of city fasttrack, then isExpresswayNext=1; Note: the start point of the following link is the node of current link; the end point is the next node point.

According to the algorithm mentioned above, we get some segment links, under the same premise of road attributes, to merger roads again by the following method, shown as Fig 3: AC: First link; CB: Second link; to calculated point $\mathrm{C}$ to the $\mathrm{AB}$ distance, if the distance lower than ROU_COMBINE_LIMIT $(5 \mathrm{~m})$, then calculate all points in $\mathrm{AC}$ and $\mathrm{CB}$ to the $\mathrm{AB}$ distance, if the distance lower than ROU_COMBINE_LIMIT $(5 \mathrm{~m})$, then AC and $\mathrm{CB}$ can be mergered into $\mathrm{AB}$.

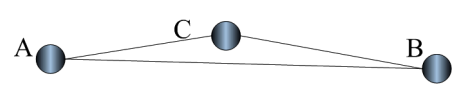

Figure 3. Road merger diagram

The starting point and end points of each road as the center, and $\mathrm{R}$ is the radius, draw a circle, $\mathrm{A}$ is the vertex of the lower left corner of the bounding rectangle of the circle which center is start point, $\mathrm{B}$ is the upper right corner vertex of the bounding rectangle of the circle which center is end pint, the bounding rectangle is the retrieval area. Shown as Fig.4, BlockLeftAx, BlockLeftAy are the lower left corner vertex, which is the code on ordinates $\mathrm{x}$, $\mathrm{y}$ direction of block file. BlockRightBx, BlockRightBy are the upper right vertex, which are the code on ordinates $\mathrm{x}$, $y$ direction of block file. SearchLeftALon, SearchLeftALat, SearchRightBLon, SearchRightBLat are the longitude and latitude of the bottom left and bottom right for retrieval rectangle range respectively. MeshBaseLeftALon, MeshBaseLeftALat are the longitude and latitude of mesh's bottom left respectively, MeshBaseLeftALon=2588048000, MeshBase LeftALat $=12288000$. The number of longitude which the width and height of BlkW, BlkH and block extends, $\mathrm{BlkW}=9216000, \mathrm{BlkHt}=6144000$.

$$
\left\{\begin{array}{l}
\text { BlockLeftAx }=(\text { SearchLeftALon }- \text { MeshBaseLeftALon }) / \text { BlkW } \\
\text { BlockLeftAy }=(\text { SearchLeftALat }- \text { MeshBaseLeftALat }) / \text { BlkH } \\
\text { BlockRightBx }=(\text { SearchRightBLon }- \text { MeshBaseLeftALon }) / \text { BlkW; } \\
\text { BlockRightBy }=(\text { SearchRightBLat }- \text { MeshBaseLeftALat }) / \text { BlkH }
\end{array}\right.
$$

Then:

$$
\left\{\begin{aligned}
\text { BlockSetNo }= & \text { Block_x / BlkDivLon } \\
& + \text { Block }_{-} y / \text { BlkDivLat } * \text { BlkSetDivLon } \\
\text { BlockNo }= & \text { Block_ } x \% \text { BlkDivLon }) \\
& +\left(\text { Block }_{-} y \% \text { usBlkDivLat }\right) * \text { BlkDivLon }
\end{aligned}\right.
$$

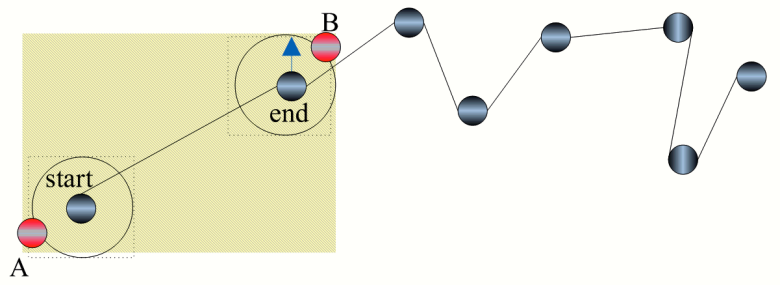

Figure 4. Determine the search range.

Such as $\operatorname{Eq}(2)$, BlockSetNo is the number of blockset where BlockSetNo locates and BlockNo is the number of blockNo where blockset locates. Block_x, Block_y are the number of block's $\mathrm{x}, \mathrm{y}$ direction respective, when block is in the Coordinate system, BlkDivLong, BlkDivLat is the segmentation magnitude of blockset, due to the blocks in blockset were separated into $8 \times 8$, so the value is 8 . BlkSetDivLong is the segmentation magnitude of blockset in mesh, so the value is 16. Algorithm that determines the range of Parcel:

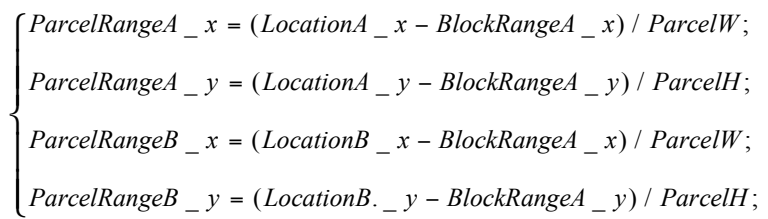

ParcelRangeA_x, ParcelRangeA_y are the number of block's top left in $\mathrm{x}, \mathrm{y}$ direction, when block was in the Mesh coordinate system. ParcelRangeB_x, ParcelRangeB_y were the number of block's top right in $\mathrm{x}$, $\mathrm{y}$ direction respective, when block was in the Mesh coordinate system. LocationA_x, LocationA_y, LocationB_x, LocationB_y are the longitude and latitude of parcel's valid range separately. BlockRangeA_x, BlockRangeA_y are the longitude and latitude of current block's bottom left. ParcelW and ParcelH are the longitude and latitude of parcel's width and height, ParcelW=288000, ParcelH=192000.

Considering the search range is the limited to certain numbers of packages and along the road, so we must consider the scale of search radius. There are five kinds of search radius for users, including $500 \mathrm{~m}, 1 \mathrm{~km}, 5 \mathrm{~km}, 10 \mathrm{~km}$, $30 \mathrm{~km}$. In the case that retrieval data is very sparse, for instance, when the search radius is not above $500 \mathrm{~m}$, then we retrieval "hospital" facilities, and if there is no result, radius will amplify to $1 \mathrm{~km}$, and retrieval again. If there is a result, then it makes a list. All the result will not stop amplifying based on this principle until $30 \mathrm{~km}$. If it doesn't finish the list within the maximum radius, then it notices the user "there is no retrieval data".

There is another case, which is that the retrieval result is extremely intensive, while the retrieval number was limited to 50, namely user only expects to see a list with 50 results. However, there are 20000 "Fast Food" within 10 kilometer in the busy zone. There are two methods to solve the problem, one is making all 20000 results when making the list index, then ranking according to the distance, picking the nearest 50. This kind of algorithm has a fatal default, which is its performance is very terrible, perhaps not acceptable by the user. Retrieval according to the original radius, if the number of retrieval results is more than 100, reduces the range of radius. Reducing the radius: 


$$
\text { The radius }=\sqrt{\frac{20 \times(\text { last radius })^{2}}{\text { number of retrieval last time }}}
$$

If the result of second times is still above 100, reuse Eq.(1) to retrieval, and so forth. Do not stop cycling until five times; display the result directly when the result number is less than 50 . If the result number is still more than 50 , then pick the nearest 50 after ranking.

\section{REALIZATION OF RETRIEVAl PROCESS}

This algorithm design two blocks of memory, one is work memory, which is used as temporarily store list index or list of all result just finished making, the other is result memory. So that the resource waste will be avoiding, and result memory will update at the appropriate time. This appropriate time is when finished making list, the list result occupies too much space, and affects GPS's performance, while UI can only display 7 records at a time, if it continue reserve the rest of the content will cause the waste of space, so result memory formulate it can only store 200 records. When the user slides the scrollbar, Layer01can represent UI to send the request of providing list, and can provide index information of $n$ to $n+7$ records meanwhile. We just need to extract them from the result memory, and then deliver to UI. When the user slides the scrollbar again, it works the same. It won't make the list again until $\mathrm{n}$ is greater than 200 .

The process of retrieval is displayed as following Fig.5. The most important part is making list index, in the whole process. Result memory, which is used to store the content of index and list at last. Namely, when we finish making index, it will copy the index list from working memory to result memory. Later working memory will be released.

\section{PERformance TeSt FOR RETRIEVAL}

The realization of retrieval along the road is computing the rectangle that we used with the utilization of each paragraph link of path, and looking for record in the parcel. The main factors which affect the retrieval performance conclude the number of link, the performance of reading parcel and the size of retrieval range. Take Shanghai as an example. Parcel range of statistical see table 1 . We could assume that the size of a standard parcel is $3.0 \times 0.8(\mathrm{~km})$. Adding printing system time statement after and behind the function of reading parcel file:

t1 = Lib_getSystemTime () ;

_read parcel file

t2 = Lib_getSystemTime();

ptprintf("\n+++ read parcel file time:\%d $\backslash \mathrm{n} ", \mathrm{t} 2-\mathrm{t} 1)$;

TABLE I.

The Statistics of ParCel Range is Displayed as Following

\begin{tabular}{cc}
\hline Place & Shanghai \\
\hline Latitude & 287106880 \\
Meter & 2979 \\
Longitude & 1119079680 \\
Meter & 845 \\
Parcel & $2979 \times 845(3.0 \times 0.8)$ \\
\hline
\end{tabular}

The result of text is the time of reading parcel and the time is about $135 \sim 42 \mathrm{~ms}$.

The number of link is displayed as following table 2 . Based on the above data, the peek value of parcel occupied by one paragraph of link is four parcels, and then the peak value of time is $4 \times 135=540 \mathrm{~ms}$. The average number of ling is 10 . So the time peak value of retrieval along the road could be 5-6s.

Testing tool: the test method is white-box texting, and realized through printing statement.

Adding printing log statement after and behind the realization of Layer03 retrieval along the road function.

time1 = Lib_getSystemTime ();

_.Make all List_

time2 = Lib_getSystemTime();

ptprintf("\n+++NOTE make list time:\%d $\backslash n$ ",t2-t1);

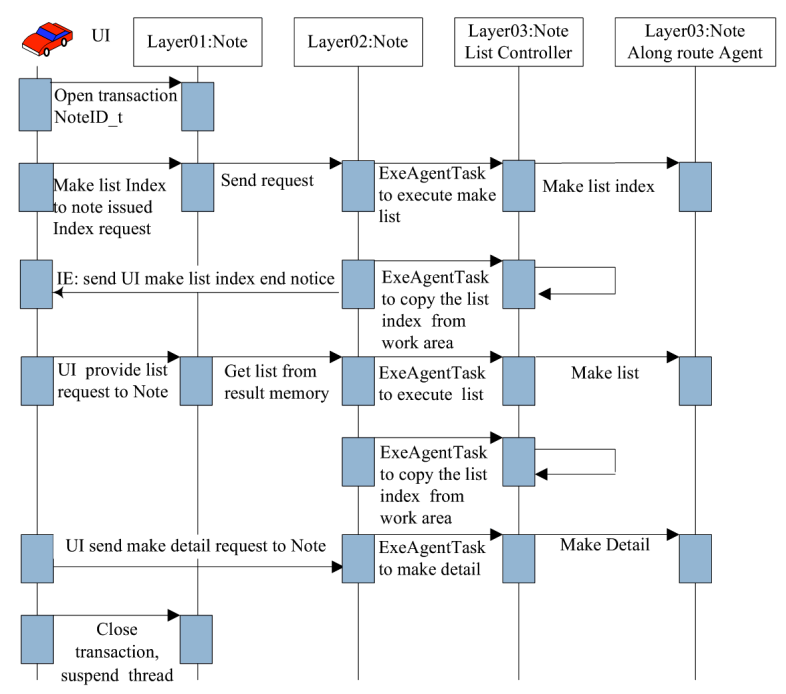

Figure 5. Retrieval time diagram.

TABLE II.

LiNK NUMBER OF THE STATISTICS

\begin{tabular}{ccc}
\hline Destination & Route distance & Link number \\
\hline Security gas stations & $35 \mathrm{~km}$ & 10 \\
Aba pickled fish & $36 \mathrm{~km}$ & 10 \\
Adon restaurant & $37 \mathrm{~km}$ & 10 \\
\hline
\end{tabular}

Note: Car position-- near the Wang hai tennis club.

Test 33 routines. The optimization of data format and radius as well as code structure brings about the enhancement of system performance. Fig. 6 a broken line graph is displayed to compare the advantage of retrieval.

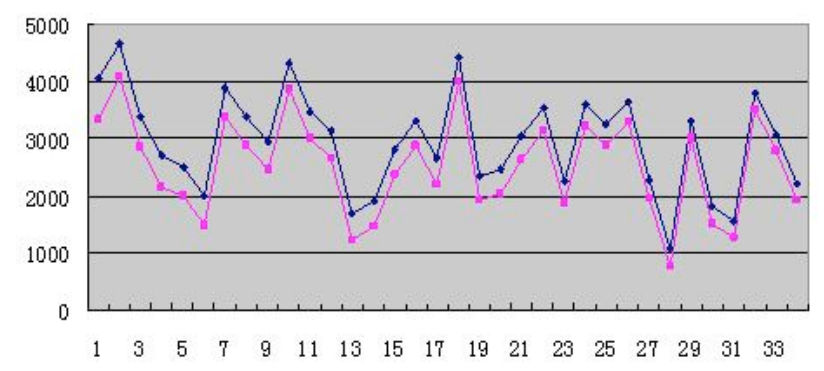

Figure 6. The comparison of before and after improvement. 


\section{CONCLUSIONS}

In recent years, vehicle navigation system has obtained the rapid development. Beginning with the status of the data retrieval system in the whole navigation, I design the modular structure of retrieval system. The whole system is divided into four modules---Layer01, Layer02, Layer03 and Layer99. Layer01 is the only external interface, is the interaction channel between NOTE and UI; Layer02 calls the lower layer interface which is the core of NOTE controlling and scheduling; Layer03 carries out all search related functions and Mark related functions; Layer99 is the common function library of NOTE, which provides NOTE's other Layer with file operational function, data access function, analytic function, transition function and ranking function. Then, the article improves the data store structure, which use B-Tree + tabular data structure, and take advantage of B-Tree to storage and manage the information index structure, tabulation is used to storage data, improves the speed of search. Then designs the whole retrieval algorithm along the road, and achieves it. In the algorithm, a new merging algorithm is proposed. The retrieval is divided into make list index, make list, make detail three steps. The algorithm puts forward a new idea that zoom the retrieval radius. Finally, the article gives the performance analysis and test results of the algorithm. As the result, the proposed algorithm can not only achieve its function, but also the high efficiency.

\section{REFERENCES}

[1] Shi Junyong, and Zhang Xiaoyu, "Study and Implementation of Real-Time Traffic Information Navigation Terminal Based on GPRS”, Computer Technology and Development, Vol.9, No.2, 2011, pp. 171-174.

[2] Huang Jing, Zhang Lin, Ji Kan, and Li Wenlong, "GPRS Traffic Statistic System on Android Platform", Computer Systems \& Applications, No.12, 2011, pp. 141-143.

[3] Zheng Rui, and Chen Jie, "New Algorithm of GPS Positioning in Incomplete Condition of Temporal Insufficient Signal", Journal of University of Electronic Science and Technology of China, Vol.34, No.4, 2009, pp. 496-500.

[4] Yang Xinqing, Design and Implementation of a Navigation Sysrem for Tousim Based on Android. Jilin university, 2011.

[5] Li Guohui, Research on Vehicle Autonomous Navigation System. Harbin Institute of Technology, 2009.

[6] Lu Kewen, The FPGA Design in Vehicle Integrated Navigation System. Beijing University of Posts and Telecommunications, 2011.
[7] Xu Shilong, Li Bin, Li Zhe, and Tian Aiguo, "Analysis on High Sensitivity GPS Navigation Methods", Radio Engineering, No.10, 2010, pp. 37-39.

[8] Bruce Powel D., Developing Real-Time System with UML, Objects, Frameworks, and Patterns, China machine press, 2002.

[9] S. Parsa, and O. Bushehrian, "The Design and Implementation of a Framework for Automatic Modularization of Software Systems". The Journal of Supercomputing, No.5, 2005, pp.71-94. http://dx.doi.org/10.1007/s11227-005-0159-5

[10] Manfred Broy, A Logical Basis for Modular Software and Systems Engineering. SOFSEM'98: Theory and Practice of Inormatics. 2002, pp. 19-35.

[11] AutoNavi Holdings Limited, A road combined methods and merge device: China, CN10219312A.

[12] Lin Zhao, and Zhenguo Yuan, "GPS/DR Vehicle Integrated Navigation System Based on Central Difference Kalman Filter", Journal of Information \& Computational Science, Vol.9, No.9, 2012, pp. 2771-2779.

[13] Hui Hu, Yuan Yuan, Huan Wang, Minghua Gao, and Dan Zou, "GPS Receiver C/A Code Rapid Acquisition Technology Research", Journal of Information \& Computational Science, Vol.10, No.2, 2013, pp. 477-484.

\section{AUTHORS}

Ren Xiaokui is an associate professor and tutor of master in Liaoning Technical University, School of Electronics and Information Engineering. He does research on Communication and information system, and Communication circuit system. His research interest is in the areas of data communication and circuit system. (email:xiujuan19880209@163.com).

Li Xiujuan is the school master. She enrolled in Communication and information systems from Liaoning Technical University, Institute of Graduate. Her research interest is in the areas of Wireless communication and data processing and vehicle navigation. (e-mail: xiujuan19880209@163.com).

Ma Lirong is the school master. She enrolled in Electronic and communication engineering from Liaoning Technical University, Institute of Graduate. Her research interest is in the areas of Micro signal detection and Signal and circuit system. (e-mail: Malirong1011@163.com).

This article is an extended and modified version of a paper presented at the International Conference on Mechanical Engineering, Automation and Material Science (MEAMS2012), held 22-23 December 2012, Wuhan, China. Received 18 March 2013. Published as resubmitted by the authors 01 May 2013. 